\title{
Clinical features of pulmonary artery sarcoma: A report of three cases
}

\author{
GUANGFA ZHU ${ }^{1}$, XIN PU ${ }^{2}$, HONGJUANG GUO ${ }^{1}$, XIAOYONG HUANG ${ }^{2}$, DONG CHEN ${ }^{3}$ and HUILI GAN $^{4}$ \\ Departments of ${ }^{1}$ Respiratory and Critical Care Medicine, ${ }^{2}$ Image Diagnosing, ${ }^{3}$ Pathology and ${ }^{4}$ Cardiac Surgery, \\ Beijing Anzhen Hospital, Capital Medical University, Beijing Institute of Heart, \\ Lung and Blood Vessel Diseases, Beijing 100029, P.R. China
}

Received February 1, 2015; Accepted March 17, 2016

DOI: $10.3892 /$ etm.2016.3408

\begin{abstract}
Pulmonary artery sarcoma (PAS) is a rare and highly malignant tumor of pulmonary artery origin. Since 1923, when the first case was reported, <300 cases have been reported worldwide. PAS has a poor prognosis, and early diagnosis with radical surgical resection offers patients with PAS the only chance of survival. However, due to its rarity and the non-specificity of its clinical manifestations and imaging presentation, PAS is frequently misdiagnosed as a pulmonary thromboembolic disease, including pulmonary thromboembolism (PTE) and chronic thromboembolic pulmonary hypertension (CTEPH). The present study reports three cases of PAS that were initially misdiagnosed as PTE or CTEPH, and were later shown to be PAS following surgery. In addition, the clinical features of these patients are examined in order to improve the differential diagnosis of PAS during the early stages of the disease, when the prognosis of patients with PAS is at its optimum.
\end{abstract}

\section{Introduction}

Pulmonary artery sarcoma (PAS) is a rare malignant tumor originating from the pulmonary blood vessels (1). Since it was initially reported by Mandelstamm (2) in 1923,<300 cases have been reported in the literature $(3,4)$. Clinically, PAS can easily be misdiagnosed as a pulmonary thromboembolic disease, such as pulmonary thromboembolism (PTE) or chronic thromboembolic pulmonary hypertension (CTEPH) (5-7). In such instances, patients lose the opportunity for early diagnosis and treatment $(8,9)$. In the present study, the clinical data of

Correspondence to: Dr Guangfa Zhu, Department of Respiratory and Critical Care Medicine, Beijing Anzhen Hospital, Capital Medical University, Beijing Institute of Heart, Lung and Blood Vessel Diseases, 2 Anzhen Road, Chaoyang, Beijing 100029, P.R. China

E-mail: guangfazhu@126.com

Key words: pulmonary artery sarcoma, pulmonary thromboembolism, differential diagnosis, computed tomography three patients with PAS, as confirmed by surgery at the Beijing Anzhen Hospital (Beijing, China), are reported and analyzed, in order to raise awareness and improve the diagnosis of PAS.

\section{Case report}

Case 1: Female patient, 36 years old. The patient was hospitalized on the 26th October 2008 having experienced shortness of breath and chest tightness for 50 days and four syncopes. In September 2007, the patient had suddenly fainted during agricultural work and had experienced loss of consciousness and incontinence for $\sim 10 \mathrm{~min}$; however, no abnormalities were detected by the local hospital upon waking. Thereafter, the patient experienced recurrent shortness of breath, chest tightness and difficulty breathing, and discontinuous syncopes occurred three times in the absence of chest pain, hemoptysis and convulsions. A chest X-ray obtained from a local hospital showed no abnormalities, although a chest computed tomography pulmonary angiography (CTPA) performed in September 2007 showed a suspected pulmonary embolism. The patient was transferred to the Beijing Anzhen Hospital to confirm the diagnosis and provide effective treatment. Prior to these events, the patient had been healthy, with no history of a rash, joint pain, lower extremity swelling or trauma surgery, and no history of oral contraceptive use.

The results of a physical examination were as follows: Blood pressure (Bp), 110/66 $\mathrm{mmHg}$ (normal range, 90-140/60-90 $\mathrm{mmHg})$; respiratory rate $(\mathrm{R})$, $20 \mathrm{times} / \mathrm{min}$ (normal range, 16-20 times/min); and heart rate (HR), 79 beats/min (normal range, 60-100 beats/min). In addition, the lung breath sounds were clear, the patient's breath did not smell and there were neither wet nor dry rales. Furthermore, the heart rhythm was regular and pulmonary valve section two (P2) heart sound hyperthyroidism and bilateral lower extremity edema were observed. The results of the laboratory tests (Automatic Blood Cell Analyzer; Sysmex XE2100; Sysmex Medical Electronics Co., Ltd., Kobe, Japan) were as follows: White blood cells (WBC), 7.1x10 $/ 1$ (normal

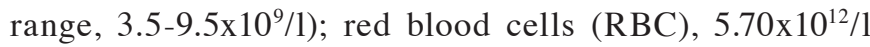

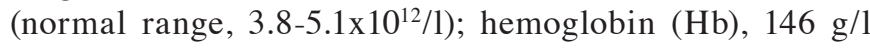
(normal range, $115-150 \mathrm{~g} / \mathrm{l}$ ); platelets (BPC), 134×10\%/1 (normal range, 125-350x10 $/ 1$ ); and D-dimer, $270 \mathrm{mg} / 1$ (normal range, 0-230 mg/1; Dimertest Latex kit; Shanghai Boatman Biotech 

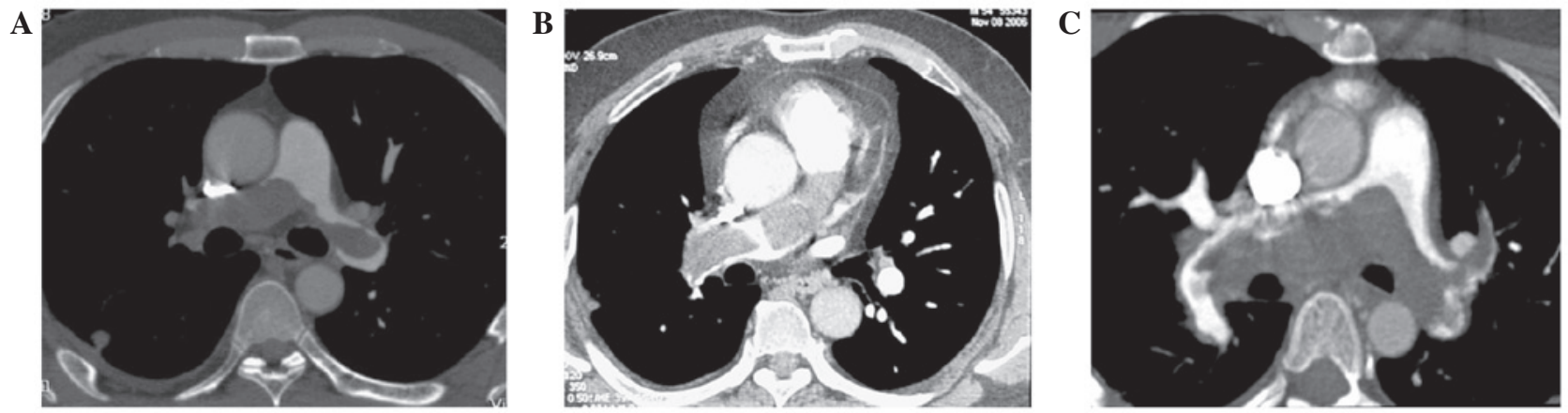

Figure 1. Computed tomography (CT) angiography of the three cases. (A) CT angiography of Case 1 showed large filling defects in the main pulmonary artery, extending to the left and the right pulmonary arteries; the right pulmonary artery was nearly obliterated. The surface of the lesion was lobulated, and the proximal end of the lesion protruded towards the right ventricular outflow tract. Both walls of the right pulmonary artery were not clearly observed (B) CT angiography of Case 2 detected large filling defects blocking the right pulmonary artery. The surface of the lesion was lobulated and segregated, with slight contrast enhancement in the venous phase. (C) CT angiography of Case 3 showed massive saddle-shaped filling defects in the main pulmonary trunk and bifurcation of the right and left pulmonary arteries. The lesion was uneven in density, had lobulated margins and protruded towards the right ventricular outflow tract. The posterior walls of the right and left pulmonary arteries were not clearly observed.

Co., Ltd., Shanghai, China). Normal results were obtained in the routine examination of stools and urine. The following results were obtained in the arterial blood gas analysis (FlexQ ABL800; Radiometer ApS, Copenhagen, Denmark): $\mathrm{pH} 7.36$ (normal range, $\mathrm{pH}$ 7.35-7.45); arterial partial pressure of carbon dioxide $\left(\mathrm{PaCO}_{2}\right), 36.5 \mathrm{mmHg}$ (normal range, $35-45 \mathrm{mmHg})$; and arterial partial pressure of oxygen $\left(\mathrm{PaO}_{2}\right)$, $80.7 \mathrm{mmHg}$ (normal range, $83-108 \mathrm{mmHg}$ ). The results of biochemical tests (Automatic Biochemical Analysis System; Beckman Coulter AU5421; Beckman Coulter, Inc., Bream CA, USA) were: Alanine aminotransferase (ALT), 50 U/1 (normal range, 7-45 U/1); aspartate aminotransferase (AST), $32 \mathrm{U} / 1$ (normal range, 13-40 g/l); total protein (TP), $68 \mathrm{~g} / 1$ (normal range, 65-85 g/l); albumin, $38 \mathrm{~g} / 1$ (normal range, 40-55 g/l); urea nitrogen, $4.5 \mathrm{mmol} / 1$ (normal range, $2.8-7.2 \mathrm{mmol} / \mathrm{l}$ ); creatinine, $79.6 \mu \mathrm{mol} / 1$ (normal range, $41-81 \mu \mathrm{mol} / \mathrm{l}$ ); lactate dehydrogenase, 233 U/1 (normal range, 140-271 U/1); creatine kinase, $37 \mathrm{U} / 1$ (normal range, 26-140 U/1); creatine kinase-MB, $12 \mathrm{U} / 1$ (normal range, 0-25 U/l); and blood glucose, $5.9 \mathrm{mmol} / \mathrm{l}$ (normal range, 3.9-6.1 mmol/1). In addition, lipid blood levels were as follows: Total cholesterol, $4.2 \mathrm{mmol} / 1$ (normal range, 3.1-5.2 mmol/1); low-density lipoprotein, $2.19 \mathrm{mmol} / 1$ (normal range, $<3.12 \mathrm{mmol} / 1$ in healthy individuals; $<2.59 \mathrm{mmol} / \mathrm{L}$ for high-risk individuals); triglyceride, $1.1 \mathrm{mmol} / 1$ (normal range, 0-1.7 mmol/l). Furthermore, tests for antinuclear antibodies (ANA) and anti-extractable nuclear antigen antibodies (ENA) were performed as previously described (10), and were negative. An electrocardiogram (ECG) showed an S1Q3T3 pattern and V1-4 T-wave inversion, and a Color Doppler vascular ultrasound, and ultrasound examinations of the liver, gall bladder, spleen and kidneys, showed that the inferior vena cava and pelvic and abdominal regions were normal. However, a Color Doppler echocardiography (UCG) showed that the diameter of the right ventricle was at the upper limit of normal (normal range, 10-20 mm) due to tricuspid aortic valve insufficiency. The pulmonary artery systolic pressure (SPAP) was $66 \mathrm{mmHg}$ (normal range, $<35 \mathrm{mmHg}$ ) and the diameter of the main pulmonary artery was $33.7 \mathrm{~mm}$ (normal range, $<27 \mathrm{~mm}$ ). A mass-like echo, with an irregular shape and uneven internal echo, was detected in the main pulmonary artery and the left pulmonary artery. The left ventricular posterior wall of the pericardial fluid areas had a depth of $\sim 8.0 \mathrm{~mm}$. A pulmonary ventilation/perfusion scan (lung V/Q) showed that all the segments of the left and right upper lung lobe possessed perfusion defects, but the ventilation was normal. Dual-color Doppler ultrasound examination revealed no abnormalities in the deep veins of the two legs. A second CTPA performed in October 2008 detected a large filling defect in the left and the right pulmonary arteries, right pulmonary artery subtotal occlusion, lobulated margins, and the pulmonary wall was less clearly observed (Fig. 1A).

On the basis of all these results, an initial diagnosis of pulmonary thromboembolism was made. Given that the patient had a history of pulmonary thromboembolism for $>50$ days (the optimum thrombolytic time) and a large embolization, the clinicians decided to perform surgical resection (pulmonary thrombus removal surgery and an embolectomy), following a discussion with the cardiac surgeons. Intraoperatively, it was observed that the mucinous tumor tissues contained the main pulmonary artery and the left and right pulmonary arteries. In addition, lesions in the posterior wall of the right pulmonary artery had infiltrated to the outside of its posterior wall and affected the nearby pericardium, and there was hemorrhagic pericardial effusion. The size (length $\mathrm{x}$ diameter) of the resected tumor tissue was $6.3 \times 2.5 \mathrm{~cm}^{2}$. Histopathological examination was performed and pathological diagnosis was PAS (type, pulmonary artery intimal sarcoma) according World Health Organization Classification of Tumors (11). Specimens were fixed in $4 \%$ neutral formalin, embedded in paraffin, sectioned to a thickness of $4 \mu \mathrm{m}$ and stained with hematoxylin and eosin (Beijing Yili Fine Chemical Co., Ltd., Beijing, China). A microscope (DM3000; Leica Microsystems, Inc., Buffalo Grove, IL, USA) was used for histopathologic analysis. Due to a weak body constitution, no chemotherapy nor radiotherapy was administered and, after 5 months, the patient succumbed to tumor recurrence and metastasis.

Case 2: Female patient, 41 years old. The patient was hospitalized on the 6th October 2009 with a 4-year history of intermittent shortness of breath and palpitations, which were relieved by rest and accompanied by abdominal distension, 
lower extremity edema, hemoptysis, chest pain, fainting, and the absence of joint swelling, pain and fever. A UCG showed that the right side of the heart was enlarged and detected pulmonary hypertension, which were considered clinical manifestations of CTEPH, right ventricular failure and respiratory failure. After receiving oxygen and anticoagulation treatment, including $0.6 \mathrm{~g}$ Clexane subcutaneously injected every 12 h for 7 days (Sanofi Winthrop Industrie, Paris, France), followed by $4.5 \mathrm{mg}$ oral administration warfarin sodium (Orion Corporation, Espoo, Finland) daily for 1 month, these symptoms were marginally relieved. Following discharge, the patient self-administered oral warfarin and hydrochlorothiazide $(25 \mathrm{mg}$ orally administrated twice daily for $>2$ years; TianJin LiSheng Pharmaceutical Co., Ltd., Tianjin, China) prior to being transferred to the Beijing Anzhen Hospital for further treatment due to aggravating and persistent symptoms, loss of appetite, fatigue and weight loss. Prior to these events, the patient had been healthy with no history of trauma surgery nor diet pill or contraceptive use. In addition, the patient had given birth to a healthy child.

The results of a physical examination were as follows: $\mathrm{T}, 36.4^{\circ} \mathrm{C}$ (normal range, $36.3-37.2^{\circ} \mathrm{C}$ ); $\mathrm{Bp}, 96 / 60 \mathrm{mmHg}$ (normal range, 90-140/60-90 $\mathrm{mmHg}$ ); HR, 80 beats/min (normal range, 60-100 beats/min); detection of a skin rash and bleeding, the lung breath sounds were clear, the patient's breath did not smell, and wet and dry rales were not detected; the heart rhythm was regular; $\mathrm{P} 2$ hyperactivity; the abdomen was soft without tenderness; there was no swelling of the liver and spleen; and edema of the lower extremities was observed. The results of laboratory tests were as follows: $\mathrm{WBC}, 6.1 \times 10^{9} / 1$; $\mathrm{RBC}, 6.3 \times 10^{12} / \mathrm{l}$; Hb, $155 \mathrm{~g} / \mathrm{l}$; BPC, 159 $\times 10^{9} / 1$; and D-dimer, $350 \mathrm{mg} / \mathrm{l}$. The results of the arterial blood gas analysis were as follows: $\mathrm{pH}$ 7.40; $\mathrm{PaCO}_{2}, 33 \mathrm{mmHg} ; \mathrm{PaO}_{2}, 61 \mathrm{mmHg}$; and N-terminal pro-brain natriuretic peptide (NT-proBNP), $2904 \mathrm{~mol} / \mathrm{l}$ (normal, <500 mol/l). An X-ray showed double lung markings, small streak shadows in the lower lobe of the left lung and thickening of the right main pulmonary artery. UCG analysis showed an enlarged right ventricle, tricuspid aortic calve insufficiency and a SPAP of $90 \mathrm{mmHg}$ (normal range, $<35 \mathrm{mmHg}$ ). CTPA detected a large block within the right pulmonary artery filling defects, lobulated margins and subtotal occlusion of the right pulmonary artery (Fig. 1B). There was no significant obstruction observed in the bilateral lower extremity vein, inferior vena cava and bilateral renal vein by Color Doppler sonography analysis.

The patient was initially diagnosed with CTEPH, and a pulmonary thromboendarterectomy was performed on the 11th October 2009. Intraoperatively, it was observed that the tumor tissue was located in the main and right pulmonary arteries; the right upper lobe pulmonary artery was entirely occluded. The size of the resected tumor tissue was $5.0 \times 1.2 \mathrm{~cm}^{2}$. The tumor was diagnosed as PAS (type, leiomyosarcoma) by a pathological diagnosis. At 1 month after the surgical procedure, the patient succumbed to septic shock and heart and lung failure in the hospital.

Case 3: Male patient, 47 years old. The patient was hospitalized on 15th July 2012 due to a 3-month history of shortness of breath during activity and fatigue, in the absence of chest palpitations, chest pain, syncopes and fever. The patient was diagnosed with a pulmonary embolism in a local hospital. The patient received local thrombolysis (Urokinase, $4400 \mathrm{IU} / \mathrm{kg}$, injection; Sichuan Bei Ao Biological Pharmaceutical Co., Ltd., Chengdu, China) via right heart catheterization followed by anticoagulation treatment (Fraxiparine, $0.4 \mathrm{mg}$ subcutaneously injected every $12 \mathrm{~h}$ for 5 days; nadroparin calcium injection, GlaxoSmithKline, Maharashtra, India; followed by warfarin sodium, $4.5 \mathrm{mg}$ oral administration daily for 1 month, Orion Corporation). Following discharge, the patient self-administered oral warfarin $(3 \mathrm{mg}$ daily for $>2$ months) and was transferred to the Beijing Anzhen Hospital for further treatment due to insignificant improvement of symptoms. From the onset the patient exhibited symptoms of anorexia and weight loss. The patient had previously been healthy, with no history of alcohol addiction, leg swelling, surgery or a family history of carcinoma.

The results of a physical examination were as follows: No cyanosis of the lips, clear breath sounds, the patient's breath did not smell, and wet and dry rales were not observed; HR, 68 times/min; P2 no hyperthyroidism; no pathological murmurs in the valve area; a soft abdomen; no swelling of the liver and spleen; ascites were negative; and no swelling of the lower limbs. The results of the laboratory tests were

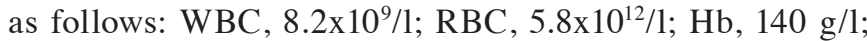
BPC, $132 \times 10^{9} / 1$; anti-human immunodeficiency virus (HIV) antibody, negative; and D-dimer, $170 \mathrm{mg} / \mathrm{l}$. The results of the arterial blood gas analysis were as follows: $\mathrm{pH}$ 7.46; $\mathrm{PaCO}_{2}$, $43 \mathrm{mmHg}$; and $\mathrm{PaO}_{2}, 83 \mathrm{mmHg}$. Biochemical tests detected no abnormalities. The prothrombin time was $35.6 \mathrm{sec}$ (normal range, 11-14 sec). An ECG showed right ventricular hypertrophy and II, III and aVF lead T-wave inversion. An X-ray showed heavy markings in the left lung and light markings in the right lung. UCG showed an enlarged right atrium and right ventricle, and a SPAP of $53 \mathrm{mmHg}$. CTPA detected a filling defect in the main, left and right pulmonary arteries. The lesion was uneven in density, had lobulated margins and protruded towards the right ventricular outflow tract. The posterior walls of the right and left pulmonary arteries were not clearly observed (Fig. 1C). A lung V/Q scan showed the bilateral iliac veins and two venous imaging and detected no abnormalities, and mismatch of lung perfusion and ventilation imaging. An abdominal B ultrasound examination detected the presence of gallstones but no abnormalities in the remaining organs of the abdominal cavity.

From these results, the patient was diagnosed with CTEPH and gallstone disease. A pulmonary thromboendarterectomy was performed on the 18th July 2012. Intraoperatively, it was shown that the tumor tissue was located in the main, left and right pulmonary arteries. The size of the resected tumor was $7.0 \times 2.7 \mathrm{~cm}^{2}$. The tumor was diagnosed as PAS (type, leiomyosarcoma) by a pathological diagnosis. The patient was transferred from Beijing Cancer Hospital (Beijing, China) on the 29th June to the Cancer Hospital for chemotherapy (50 $\mathrm{mg}$ doxorubicin and $5 \mathrm{~g}$ ifosfamide every 3 weeks for six cycles). However, the patient succumbed to tumor recurrence and metastasis after 18 months.

The present study was conducted in accordance with the declaration of Helsinki and with approval from the Ethics Committee of the Beijing Anzhen Hospital. Written informed consent was obtained from all participants. 


\section{Discussion}

PAS is a primary tumor that occurs in the pulmonary valve and/or pulmonary artery $(1,12)$. The diagnosis of PAS must rule out tumor metastases from other parts of the body (13). The incidence of PAS worldwide ranges from 0.001 to $0.03 \%$; however, the underlying pathogenesis of PAS remains unclear (14). The ages of individuals previously diagnosed with PAS range from 13-86 years, with an average age of 52-years-old, and the ratio of females to males is 2:1 (15). PAS may be divided into numerous pathological types, according to 138 cases of pathological specimens summarized in the literature $(14,16,17)$; these types include pulmonary artery intimal sarcoma (31.2\%), leiomyosarcoma (15.9\%), spindle cell sarcoma (13.8\%), malignant fibrous tissue sarcoma (7.2\%), fibrosarcoma (5.1\%), fibromyxoid sarcoma (4.3\%), rhabdomyosarcoma (4.3\%) and chondrosarcoma (3.6\%) (16). The present study reports the analysis of three cases of PAS, including one case of pulmonary artery intimal sarcoma and two cases of leiomyosarcoma. Due to the small number of reported cases, the incidence of the various pathological types of PAS in the Chinese population requires further study.

There are a lack of specific clinical manifestations for primary pulmonary sarcomas $(17,18)$. The majority of patients have demonstrated insidious onset, predominantly involving progressive dyspnea, chest pain, coughing, hemoptysis, syncopes, fever, fatigue and weight loss $(4,6,8,15,16)$. X-rays of these patients typically show a prominent hilar shadow, sparse texture of the peripheral vasculature, lung nodules and enlargement of the heart. A clinical diagnosis based on a CTPA has numerous benefits, and it should be used as a routine examination, which can be expressed as the pulmonary artery dilatation, intraluminal filling defect and luminal stenosis. It has been reported that $\sim 90 \%$ of PAS lesions affected at least two parts of the pulmonary arteries, $>85 \%$ of patients had primary pulmonary lesions, $71 \%$ of patients had right pulmonary artery lesions, $65 \%$ of patients had left pulmonary artery lesions, and $10 \%$ of patients had lesions involving the right ventricular outflow channel (19). In previous studies, a Color Doppler UCG showed right ventricular enlargement, tricuspid regurgitation and pulmonary hypertension $(15,20)$. The Doppler vascular ultrasound examination results of the lower limbs, inferior vena cava and pelvis were normal. Arterial blood gas analysis showed decreased $\mathrm{PaO}_{2}$ levels. Clinically, it is easy to misdiagnose the patients with PTE or CTEPH, since the clinical manifestations of pulmonary thromboembolic diseases are similar to PAS (5-7). Therefore, confirmation of the diagnosis typically requires surgery $(19,21)$.

The present study reports three cases of PAS at the Beijing Anzhen Hospital with symptoms resembling PTE. Arterial blood gas analyses, ECG characteristics, X-ray scans, and lung V/Q examination results prior to surgery misdiagnosed the patients with PTE (Case 1) or CTEPH (Cases 2 and 3). The clinical and imaging findings of the three patients were carefully analyzed and compared with the literature. From this, the authors of the present study propose the following to aid in the differential diagnosis of PAS: i) PAS exhibits insidious onset and slow progression, as compared with the rapid onset of PTE; ii) PAS patients typically show signs of a fever, loss of appetite, weight loss and other systemic manifestations; iii) PAS patients typically lack clinical manifestations that may cause pulmonary emboli, including deep vein thrombosis; iv) CTPA of PAS patients usually detects abnormalities that are typically absent from PTE patients, including filling defects in the main pulmonary artery, which may extend to the right and/or left pulmonary artery, as well as lobulated margins in the lesion or segregation phenomena, which project in the direction of blood flow in the right ventricular outflow tract; and v) PAS does not respond to thrombolysis or anticoagulation therapy, and may deteriorate after treatment. In addition, previous studies have recommended that the CT data be used together with positron-emission tomography (PET)-CT results for the diagnosis of PAS (4,7-9). The performance of ${ }^{18}$ F-fludeoxyglucose $\left({ }^{18} \mathrm{FDG}\right) \mathrm{PET}-\mathrm{CT}$ in patients with PTE or CTEPH showed no increase in radiotracer intake, whereas in patients with PAS, ${ }^{18} \mathrm{FDG}$ PET-CT showed an increase in radiotracer uptake, which may help to differentiate between the two diseases.

PAS has a poor prognosis; its natural survival period is $\sim 1.5$ months following diagnosis (15). Surgery is the preferred treatment for PAS, since it can relieve symptoms and prolong the survival period (9). Kim et al (22) successfully completed surgical resection on nine patients with PAS; after 1 month, the cardiac functions of the patients were of grade 1 (New York Heart Association Classification system) (23) and their exercise capacities were improved. Furthermore, their postoperative mean survival period was 19.2 months, including one patient who survived for $>45$ months (21). In addition, the use of chemotherapeutics combined with surgery to treat PAS has shown some efficacy; Xu et al (24) reported a patient with intimal PAS who was successfully treated with a vinorelbine-based regimen as second-line chemotherapy and was stable at 19 months following the onset of postoperative recurrence. Surgery, combined with chemotherapy and (or) radiotherapy post-surgery, may increase the survival period to 1-2 years.

In conclusion, PAS is an extremely rare tumor that occurs in the cardiovascular system. Due to its non-specific clinical manifestations, PAS is often misdiagnosed as pulmonary thromboembolic diseases. Therefore, PAS should be suspected in patients with large 'mass' within the pulmonary trunk and its main branches, and who have insidious onset, no history of venous thromboembolism and no evidence of positive hypercoagulability, particularly in those who are unresponsive to current anticoagulation therapy or in which there is clinical evidence of neoplasm. These patients should be investigated to confirm the diagnosis and should undergo surgical intervention as soon as possible, in order to improve the prognosis of PAS.

\section{References}

1. Travis WD, Brambilla E, Müller Hermelink HK and Harris CC (eds): World Health Organization Classification of Tumours. Pathology \& Genetics of Tumours of the Lung, Pleura, Thymus and Heart. IARC Press, Lyon, 2004.

2. Mandelstamm M: About primary neoplasms of the heart. Virchows Arch A Pathol Pathol Anat 245: 43-54, 1923 (In German).

3. Shehatha J, Saxena P, Clarke B, Dunning J and Konstantinov IE: Surgical management of extensive pulmonary artery sarcoma. Ann Thorac Surg 87: 1269-1271, 2009.

4. Gan HL, Zhang JQ, Huang XY and Yu W: The wall eclipsing sign on pulmonary artery computed tomography angiography is pathognomonic for pulmonary artery sarcoma. PLoS One 8: e83200, 2013. 
5. El-Sayed Ahmed MM, Aftab M, Al-Najjar RM, de la Cruz KI, Benjamin RS and Hallman CH: Pulmonary artery sarcoma mimicking pulmonary embolism. Tex Heart Inst J 41: 515-517, 2014.

6. Renilla A, Fernández-Vega I, Martín M and Weinsaft JW Pulmonary artery sarcoma mimicking a pulmonary embolism. Eur Heart J Cardiovasc Imaging 14: 1025, 2013.

7. Sandhu A, Yates TJ and Kuriakose P: Pulmonary artery sarcoma mimicking a pulmonary embolism. Indian J Cancer 45: 27-29, 2008.

8. Attinà D, Niro F, Tchouanté P, Mineo G, Russo V, Palazzini M, Galiè N, Fanti S, Lovato L and Zompatori M: Pulmonary artery intimal sarcoma. Problems in the differential diagnosis. Radiol Med 118: 1259-1268, 2013

9. Bhagwat K, Hallam J, Antippa P and Larobina M: Diagnostic enigma: Primary pulmonary artery sarcoma. Interact Cardiovasc Thorac Surg 14: 342-344, 2012.

10. Arbuckle MR, McClain MT, Rubertone MV, Scofield RH, Dennis GJ, James JA and Harley JB: Development of autoantibodies before the clinical onset of systemic lupus erythematosus. N Engl J Med 349: 1526-1533, 2003.

11. Fletcher CDM, Unni KK and Mertens F (eds): World Health Organization Classification of Tumours. Pathology \& genetics of tumours of soft tissue and bone. IARC Press, Lyon, 2002.

12. Fletcher CDM, Unni KK and Mertens F (eds): World Health Organization Classification of Tumours. Pathology \& Genetics of Tumours of Soft Tissue and Bone. IARC Press, Lyon, 2002.

13. Dimitrakakis G, Zilidis G, Buchalter M and Von Oppell U: Pulmonary artery sarcoma-a challenging diagnosis: A case report. Heart Surg Forum 9: E897-E899, 2006.

14. Huo L, Moran CA, Fuller GN, Gladish G and Suster S: Pulmonary artery sarcoma: A clinicopathologic and immunohistochemical study of 12 cases. Am J Clin Pathol 125: 419-424, 2006.
15. Parish JM, Rosenow EC III, Swensen SJ and Crotty TB: Pulmonary artery sarcoma-clinical features. Chest 110: 1480-1488, 1996.

16. Cox JE, Chiles C, Aquino SL, Savage P and Oaks T: Pulmonary artery sarcomas: A review of clinical and radiologic features. J Comput Assist Tomogr 21: 750-755, 1997.

17. Keel SB, Bacha E, Mark EJ, Nielsen GP, Rosenberg AE: Primary pulmonary sarcoma: A clinicopathologic study of 26 cases. Mod Pathol 12: 1124-1131, 1999.

18. Pewarchuk JA, Nassaralla CL and Midthun DE: A 39-year-old woman with cough, chest pressure, and worsening dyspnea. Chest 131: 934-937, 2007.

19. Scheffel H, Stolzmann P, Plass A, Weber A, Prêtre R, Marincek B and Alkadhi H: Primary intimal pulmonary artery sarcoma: A diagnostic challenge. J Thorac Cardiovasc Surg 135: 949-950, 2008.

20. Zurick AO 3rd, Lenge De Rosen V, Tan CD, Rodriguez ER, Flamm SD and Schoenhagen P: Pulmonary artery intimal sarcoma masquerading as pulmonary embolism. Circulation 124: 1180-1181, 2011.

21. Yi CA, Lee KS, Choe YH, Han D, Kwon OJ and Kim S: Computed tomography in pulmonary artery sarcoma: Distinguishing features from pulmonary embolic disease. J Comput Assist Tomogr 28: 34-39, 2004.

22. Kim HK, Choi YS, Kim K, Shim YM, Sung K, Lee YT, Park PW and Kim J: Surgical treatment for pulmonary artery sarcoma. Eur J Cardiothorac Surg 33: 712-716, 2008.

23. The Criteria Committee of the New York Heart Association: Nomenclature and Criteria for Diagnosis of Diseases of the Heart and Great Vessels. 9th edition. Little, Brown \& Co, Boston, MA, pp253-256, 1994.

24. Xu Y, Wang K, Geng Y, Shao Y and Yin Y: A case of intimal sarcoma of the pulmonary artery successfully treated with chemotherapy. Int J Clin Oncol 17: 522-527, 2012. 